\title{
Terbium-based extreme ultraviolet multilayers
}

\author{
David L. Windt \\ Columbia Astrophysics Laboratory, 550 West 120th Street, New York, New York 10027 \\ John F. Seely \\ Naval Research Laboratory, 4555 Overlook Avenue South West, Washington, DC 20375
}

Benjawan Kjornrattanawanich

Universities Space Research Association, National Synchrotron Light Source, Brookhaven National Laboratory, Upton, New York 11973

\author{
Yu. A. Uspenskii \\ Lebedev Physical Institute, Leninski Prospekt 53, 117924 Moscow, Russia
}

\begin{abstract}
Received July 20, 2005; revised manuscript received August 12, 2005; accepted August 16, 2005
We have fabricated periodic multilayers that comprise either $\mathrm{Si} / \mathrm{Tb}$ or $\mathrm{SiC} / \mathrm{Tb}$ bilayers, designed to operate as narrowband reflective coatings near $60 \mathrm{~nm}$ wavelength in the extreme ultraviolet (EUV). We find peak reflectance values in excess of $20 \%$ near normal incidence. The spectral bandpass of the best $\mathrm{Si} / \mathrm{Tb}$ multilayer was measured to be $6.5 \mathrm{~nm}$ full width at half-maximum (FWHM), while $\mathrm{SiC} / \mathrm{Tb}$ multilayers have a more broad response, of order $9.4 \mathrm{~nm}$ FWHM. Transmission electron microscopy analysis of $\mathrm{Si} / \mathrm{Tb}$ multilayers reveals polycrystalline $\mathrm{Tb}$ layers, amorphous Si layers, and relatively large asymmetric amorphous interlayers. Thermal annealing experiments indicate excellent stability to $100^{\circ} \mathrm{C}(1 \mathrm{~h})$ for $\mathrm{Si} / \mathrm{Tb}$. These new multilayer coatings have the potential for use in normal incidence instrumentation in a region of the EUV where efficient narrowband multilayers have not been available until now. In particular, reflective $\mathrm{Si} / \mathrm{Tb}$ multilayers can be used for solar physics applications where the coatings can be tuned to important emission lines such as $\mathrm{O} V$ near $63.0 \mathrm{~nm}$ and $\mathrm{Mg} X$ near $61.0 \mathrm{~nm}$. (c) 2005 Optical Society of America OCIS codes: $230.4170,310.1620,310.6860,340.0340,350.1260$.
\end{abstract}

Narrowband reflective multilayer coatings operating near normal incidence in the extreme ultraviolet (EUV) band have by now found wide application in a number of disciplines, including solar physics, photolithography, short-wavelength lasers, plasma diagnostics, etc. While peak reflectance values as high as $70 \%$ have been obtained at the short-wavelength end of the EUV near $13 \mathrm{~nm}$, at longer wavelengths the performance of even the best coatings is considerably lower. The limit on multilayer performance at long wavelengths is primarily due to absorption in the constituent materials; an ideal EUV multilayer would comprise materials with low absorption so that the incident radiation can penetrate deeply into the multilayer stack, thereby allowing for the coherent addition of reflections from a large number of layer interfaces. But with high-absorption materials only a few interfaces can contribute to the reflection process, thereby limiting the ultimate performance.

Our principal motivation for the development of long-wavelength EUV multilayers is directed at the production of narrowband imaging telescopes for solar physics. A number of important coronal emission lines in the 60-65 $\mathrm{nm}$ band, including $O V$ near 63.0 $\mathrm{nm}$ and $\mathrm{Mg} X$ near $61.0 \mathrm{~nm}$, could be observed if efficient narrowband multilayer coatings were available. Multilayer imagers that could be tuned to these wavelengths would nicely complement currently available multilayers that operate at shorter wavelengths, and that have been used in satellite instruments such as SoHO/EIT, ${ }^{1}$ TRACE, ${ }^{2}$ and the SDO/AIA $^{3}$ instrument currently under development, all of which have been limited to wavelengths shorter than $34 \mathrm{~nm}$.

In recent years narrowband multilayer performance has been extended to longer EUV wavelengths with materials such as $\mathrm{Mg}$ and $\mathrm{Sc}: \mathrm{SiC} / \mathrm{Mg}$ multilayers ${ }^{4}$ show high reflectance in the range 25-40 nm, i.e., below the $\mathrm{Mg} K$ edge near $25 \mathrm{~nm}$ (and will be used in the SDO/AIA instrument), while Si/Sc multilayers ${ }^{5}$ operate with relatively high efficiency in the $35-50 \mathrm{~nm}$ range, i.e., below the $3 p-3 d$ absorption "window" for this material. However, at wavelengths longer than $\sim 50 \mathrm{~nm}$ the development of efficient multilayers has been limited thus far by a dearth of suitable multilayer materials having sufficiently low absorption.

We have identified the rare-earth elements, with partially filled $5 d$ and $5 f$ levels, as candidate materials having transmission windows at wavelengths longer than $50 \mathrm{~nm}$. Tb in particular was found to have low absorption in the $60-70 \mathrm{~nm}$ range. ${ }^{6} \mathrm{The} \mathrm{Tb}$ transmission data, obtained by measuring with synchrotron radiation the transmittance of $\mathrm{Tb}$ films of varying thickness deposited directly onto Si photodiodes, ${ }^{7}$ are shown in Fig. 1 . Driven by these results we have investigated new EUV multilayers comprising $\mathrm{Si} / \mathrm{Tb}$ and $\mathrm{Sic} / \mathrm{Tb}$ bilayers designed for normal incidence reflectance in this same wavelength band. Our optical constants determination work will be described in detail elsewhere; we present our multilayer results here.

Multilayer films were deposited by dc magnetron sputtering in argon, using a system that has been de- 


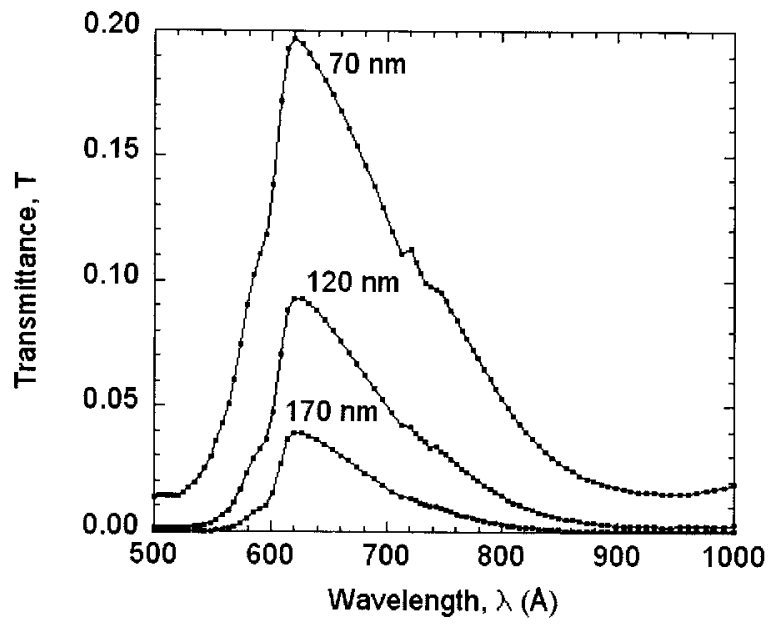

Fig. 1. Measured transmission of $\mathrm{Tb}$ films having the thicknesses indicated, as a function of wavelength.

scribed in detail previously. ${ }^{8}$ The coating system utilizes $S$-gun cathodes, which were operated in constant-power mode. The base pressure of the coating chamber was in the range $(2-3) \times 10^{-7}$ Torr. With an Ar flow rate of $\sim 6 \mathrm{sccm}$ the Ar pressure was maintained at $2.0 \mathrm{mT}$ Torr. $\mathrm{Si} / \mathrm{Tb}$ and $\mathrm{SiC} / \mathrm{Tb}$ multilayer films were grown on $16 \times 16 \mathrm{~mm} \mathrm{Si}$ wafer segments cleaved from polished 3 -in. (100) wafers. Effective deposition rates of order $0.04 \mathrm{~nm} / \mathrm{s}$ were obtained for $\mathrm{Tb}(30 \mathrm{~W}$ applied power), $0.04 \mathrm{~nm} / \mathrm{s}$ for $\mathrm{Si}(45 \mathrm{~W})$, and $0.03 \mathrm{~nm} / \mathrm{s}$ for $\mathrm{SiC}(30 \mathrm{~W})$.

Grazing incidence $\mathrm{x}$-ray reflectance (XRR) measurements were made in the $\theta-2 \theta$ geometry using a system comprising a sealed-tube $\mathrm{Cu}$ anode operating at $1.3 \mathrm{~kW}$ and a Ge crystal monochromator tuned to the $\mathrm{Cu} K \alpha$ line $(8 \mathrm{keV})$. Fits $^{9}$ to the measured XRR data were used to determine multilayer periods and the layer thickness ratio $\Gamma$, defined here as the $\mathrm{Si}$ or $\mathrm{SiC}$ layer thickness divided by the multilayer period $d$.

EUV reflectance measurements were made as a function of wavelength at $5^{\circ}$ from normal at beamline X24C at the National Synchrotron Light Source. The absolute energy scale of the grating monochromator was calibrated by measuring the Sn and In absorption edges (using filters made from these elements) at 23.97 and $16.70 \mathrm{eV}$, respectively. The absolute reflectance was computed as the reflected beam intensity divided by the incident intensity as measured with the same Si photodiode for each wavelength from roughly 49 to $80 \mathrm{~nm}$.

Preliminary reflectance calculations (made by using our measured optical constants for $\mathrm{Tb}$ and optical constants for $\mathrm{Si}$ and $\mathrm{SiC}$ from Refs. 10 and 11) suggested that the reflectance and spectral bandpass of $\mathrm{Si} / \mathrm{Tb}$ and $\mathrm{SiC} / \mathrm{Tb}$ multilayers varies predictably with $\Gamma$ such that an optimal $\Gamma$ value should exist that yields high peak reflectance and a relatively narrow spectral response. However, the experimental reflectance data obtained with a series of $\mathrm{Si} / \mathrm{Tb}$ and $\mathrm{SiC} / \mathrm{Tb}$ multilayers for which the $\Gamma$ value was varied systematically and the period adjusted to keep the peak wavelength constant - the so-called "through- $\Gamma$ series"-revealed that, while the spectral bandpass does indeed increase with $\Gamma$ as expected, the peak reflectance is relatively insensitive to $\Gamma$ variations over the range $\sim 0.2-0.6$.

$\mathrm{The} \mathrm{Si} / \mathrm{Tb}$ and $\mathrm{SiC} / \mathrm{Tb}$ films found to have the highest peak reflectance and smallest spectral bandpasses from our through- $\Gamma$ investigations are shown in Fig. 2. We find peak reflectance values of $\sim 22 \%$ at $\lambda \sim 60 \mathrm{~nm}$ for both films. The spectral bandpass of the $\mathrm{Si} / \mathrm{Tb}$ film was measured to be $6.5 \mathrm{~nm} \mathrm{FWHM}$, while $\mathrm{SiC} / \mathrm{Tb}$ multilayers have a more broad response, of order $9.4 \mathrm{~nm}$ FWHM. For films designed to operate at somewhat longer wavelengths the spectral bandpass increased further; for example, a $\mathrm{SiC} / \mathrm{Tb}$ film peaking near $67 \mathrm{~nm}$ was found to have a $12.5 \mathrm{~nm}$ FWHM bandpass.

The individual layer thicknesses for these films were estimated by fitting the measured EUV reflectance curves. We find for the $\mathrm{Si} / \mathrm{Tb}$ film having $N$ $=10$ bilayers shown in Fig. 2 a period $d=30.2 \mathrm{~nm}$ and $\Gamma=0.26$, while the $\mathrm{SiC} / \mathrm{Tb}$ film has $N=10, d$ $=31.0 \mathrm{~nm}$, and $\Gamma=0.30$. Comparing the EUV fits with equivalent fits to the XRR data reveals significant discrepancies between the $d$ and $\Gamma$ values determined from each technique. For example, in the case of the $\mathrm{Si} / \mathrm{Tb}$ film shown in Fig. 2, fixing the layer thickness ratio to $\Gamma=0.26$ (so as to be consistent with the EUV fit) the XRR data yields $d=31.8 \mathrm{~nm}$, i.e., a difference in period of $1.6 \mathrm{~nm}$ between the two techniques. Furthermore, relatively large interface widths, of order $\sigma=2.8 \mathrm{~nm}$, are required for acceptable fits to the EUV data.

The large $\sigma$ values determined from the EUV data suggest the possibility of substantial intermixing at the $\mathrm{Si}-\mathrm{Tb}$ and $\mathrm{SiC}-\mathrm{Tb}$ interfaces. This possibility has been confirmed, at least for the case of $\mathrm{Si} / \mathrm{Tb}$, by high-resolution transmission electron microscopy (TEM) analysis. Cross-sectional TEM images of selected samples were made by Accurel Systems International, ${ }^{12}$ with thinned samples prepared using a focused ion-beam technique. Shown in Fig. 3 is a TEM image of the $\mathrm{Si} / \mathrm{Tb}$ film in Fig. 2 described above. The polycrystalline and strongly textured $\mathrm{Tb}$ layers have a thickness of order $7 \mathrm{~nm}$, while the

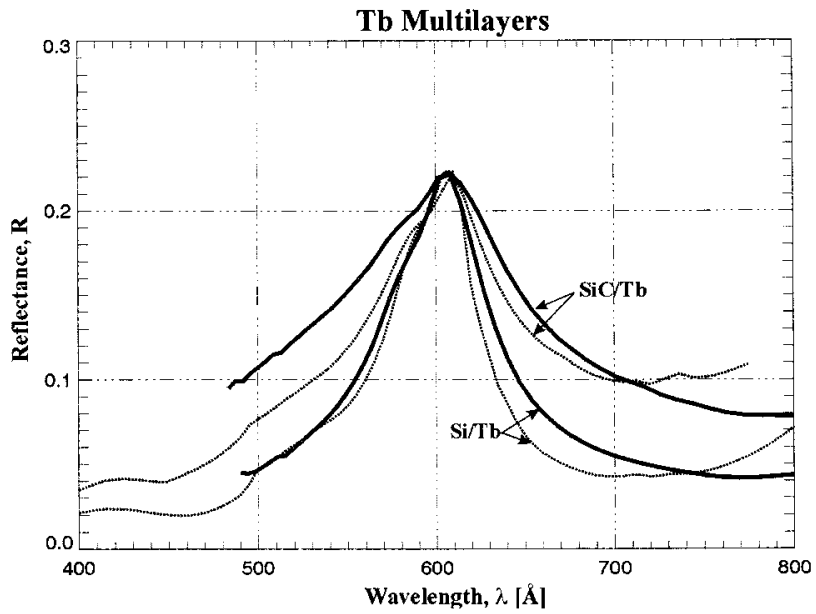

Fig. 2. Reflectance at $5^{\circ}$ incidence of $\mathrm{Si} / \mathrm{Tb}$ and $\mathrm{SiC} / \mathrm{Tb}$ multilayers as indicated. Solid lines are measured lines; dotted lines are fits. 


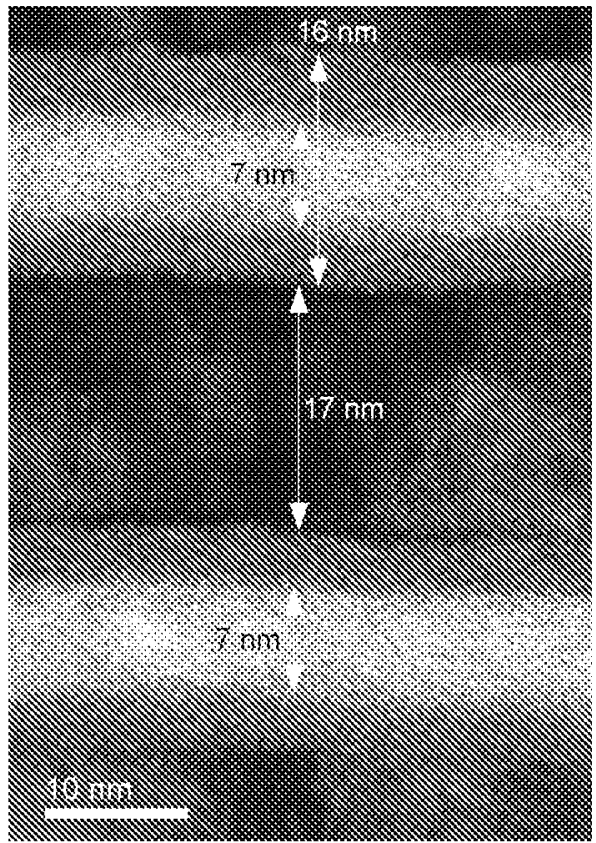

Fig. 3. High-resolution TEM image of the $\mathrm{Si} / \mathrm{Tb}$ film shown in Fig. 2.

amorphous Si layers have a thickness of $17 \mathrm{~nm}$. Asymmetric amorphous interlayers can be clearly identified in the image, with the Tb-on-Si interlayer having a thickness of order $4 \mathrm{~nm}$, while the $\mathrm{Si}-\mathrm{on}-\mathrm{Tb}$ interface is somewhat thicker, of order $5 \mathrm{~nm}$. The 33 $\mathrm{nm}$ period determined from TEM analysis is greater than the $31.8 \mathrm{~nm}$ XRR period and the $30.2 \mathrm{~nm}$ EUV period.

These discrepancies in the layer thicknesses inferred from EUV, XRR, and TEM analyses may be explained by the optical effects of the large amorphous $\mathrm{Si}-\mathrm{Tb}$ interlayers identified by TEM. That is, these interlayer regions almost certainly have optical constants that differ from either the pure $\mathrm{Si}$ or Tb layers. If the optical constants of these interlayers were known in the EUV and x-ray bands, presumably they could be included in a four-layer model to better fit the measured reflectance data. But unfortunately not even the density and exact composition of these interlayers, let alone the EUV optical constants, are known at present. Consequently, we are not able to accurately model our data and reconcile the apparent discrepancies in the fit parameters at present.

The thermal stability of $\mathrm{Si} / \mathrm{Tb}$ and $\mathrm{SiC} / \mathrm{Tb}$ multilayers was investigated by performing $1 \mathrm{~h}$ annealing in air at $100^{\circ} \mathrm{C}, 200^{\circ} \mathrm{C}$, and $300^{\circ} \mathrm{C}$ for prototype films. The annealed films were compared with the asdeposited films using both XRR and EUV reflectance measurements. We find that in the case of $\mathrm{Si} / \mathrm{Tb}$, the peak EUV reflectance was essentially unchanged after the $100^{\circ} \mathrm{C}$ anneal; however, the reflectance peak shifted by $0.2 \mathrm{~nm}$ towards shorter wavelengths (corresponding to a period decrease of $\sim 0.1 \mathrm{~nm}$ ). After the $200^{\circ} \mathrm{C}$ anneal the reflectance showed a relative decrease of $10 \%$, and the reflectance peak shifted by another $1.1 \mathrm{~nm}$. The XRR data were consistent with these period changes, and further indicated that after the $300^{\circ} \mathrm{C}$ anneal the multilayer structure had changed drastically; the EUV reflectance of the $300^{\circ} \mathrm{C}$ annealed film was not measured.

In the case of $\mathrm{SiC} / \mathrm{Tb}$ films, much smaller period shifts (less than $0.1 \mathrm{~nm}$ ) were identified after thermal annealing to $200^{\circ} \mathrm{C}$. However, a $5 \%$ relative decrease in peak EUV reflectance was measured after the $100^{\circ} \mathrm{C}$ anneal, and another $5 \%$ decrease was measured after the $200^{\circ} \mathrm{C}$ anneal. Again, because of the drastic change in period after the $300^{\circ} \mathrm{C}$ anneal as inferred from XRR measurements, the EUV reflectance was not measured for this sample.

The combination of relatively high peak reflectance (22\%), narrow spectral response (6.5 nm FWHM), and good thermal stability to $100^{\circ} \mathrm{C}$ of the $\mathrm{Si} / \mathrm{Tb}$ multilayer shown in Figs. 2 and 3 make this coating an attractive candidate for a narrowband imager tuned near $60 \mathrm{~nm}$. For solar physics applications additional suppression of nearby emission lines will likely be necessary as well, to achieve sufficient spectral "purity." For this purpose Tb thin-film filters currently under study may provide a viable solution when used in conjunction with reflective $\mathrm{Si} / \mathrm{Tb}$ multilayers. Looking to the future, the possibility of diffusion barrier layers deposited between the $\mathrm{Si}$ and $\mathrm{Tb}$ layers (as has been already demonstrated, e.g., in $\mathrm{Si} / \mathrm{W} / \mathrm{Sc}$ multilayers ${ }^{13}$ ), might make it possible to achieve even higher peak reflectance and greater spectral selectivity; the use of such diffusion barrier layers is currently under study.

This research was supported by NASA Grant No. NNH04AA13I. D. Windt's e-mail address is windt@astro.columbia.edu.

\section{References}

1. J. P. Delaboudinière and the SOHO Mission groups, Sol. Phys. 162, 291 (1995).

2. B. N. Handy, L. W. Acton, C. C. Kankelborg, and 43 coauthors, Sol. Phys. 187, 229 (1999).

3. R. Soufli, D. L. Windt, J. C. Robinson, S. L. Baker, A. L. Aquila, F. J. Dollar, E. M. Gullikson, J. F. Seely, B. Kjornrattanawanich, and L. Golub, Proc. SPIE $\mathbf{5 9 0 0}$ (2005).

4. T. Ejima, Y. Kondo, and M. Watanabe, Jpn. J. Appl. Phys., Part 1 40, 376 (2001).

5. Yu. A. Uspenskii, V. E. Levashov, A. V. Vinogradov, A. I. Fedorenko, V. V. Kondratenko, Yu. P. Pershin, E. N. Zubarev, and V. Yu. Fedotov, Opt. Lett. 23, 771 (1998).

6. J. F Seely, L. Goray, D. Windt, B. Kjornrattanawanich, Y. Uspenskii, and A. Vinogradov, Proc. SPIE 5538, 43 (2004).

7. Yu. A. Uspenskii, J. F. Seely, N. L. Popov, A. V. Vinogradov, Yu. P. Pershin, and V. V. Kondratenko, J. Opt. Soc. Am. A 21, 298 (2004).

8. J. Dalla Torre, G. H. Gilmer, D. L. Windt, R. Kalyanaraman, F. H. Bauman, P. L. O'Sullivan, J. Sapjeta, T. Diaz de la Rubia, and M. Djafari Rouhani, J. Appl. Phys. 94, 263 (2003).

9. D. L. Windt, Comput. Phys. 12, 360 (1998).

10. B. L. Henke, E. M. Gullikson, and J. C. Davis, At. Data Nucl. Data Tables 54, 181 (1993).

11. J. B. Kortright and D. L. Windt, Appl. Opt. 27, 2841 (1988).

12. Accurel Systems International, Sunnyvale, Calif.

13. J. F. Seely, Yu. A. Uspenskii, Yu. P. Pershin, V. V. Kondratenko, and A. V. Vinogradov, Appl. Opt. 41, 1846 (2002). 